\title{
Heterogeneous ligand-free rhodium oxide catalyst embedded within zeolitic microchannel to enhance regioselectivity in hydroformylation
}

Cun Liu, Jingjing Zhang, Haiou Liu, Jieshan Qiu, Xiongfu Zhang*

\author{
State Key Laboratory of Fine Chemicals, School of Chemical Engineering, \\ Dalian University of Technology, Dalian, 116024, China
}

*Corresponding author: Tel/Fax: +86 411 84986155; E-mail: xfzhang@dlut.edu.cn (X. F. Zhang) 


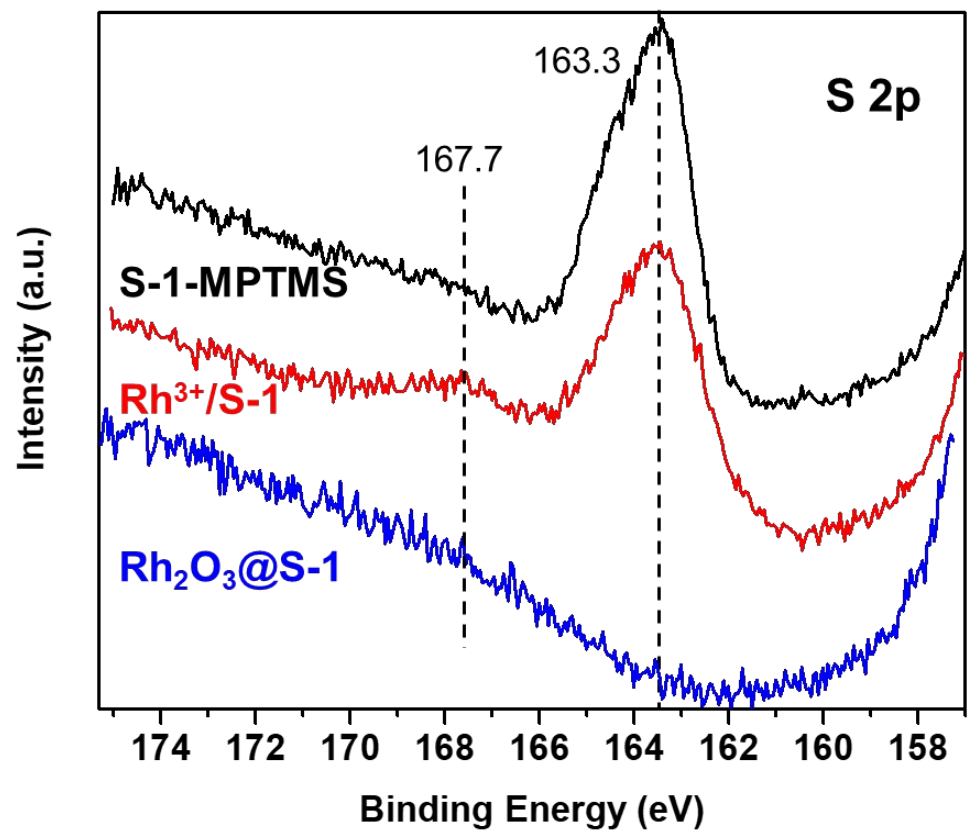

Figure S1. S 2P XPS spectra of different samples.

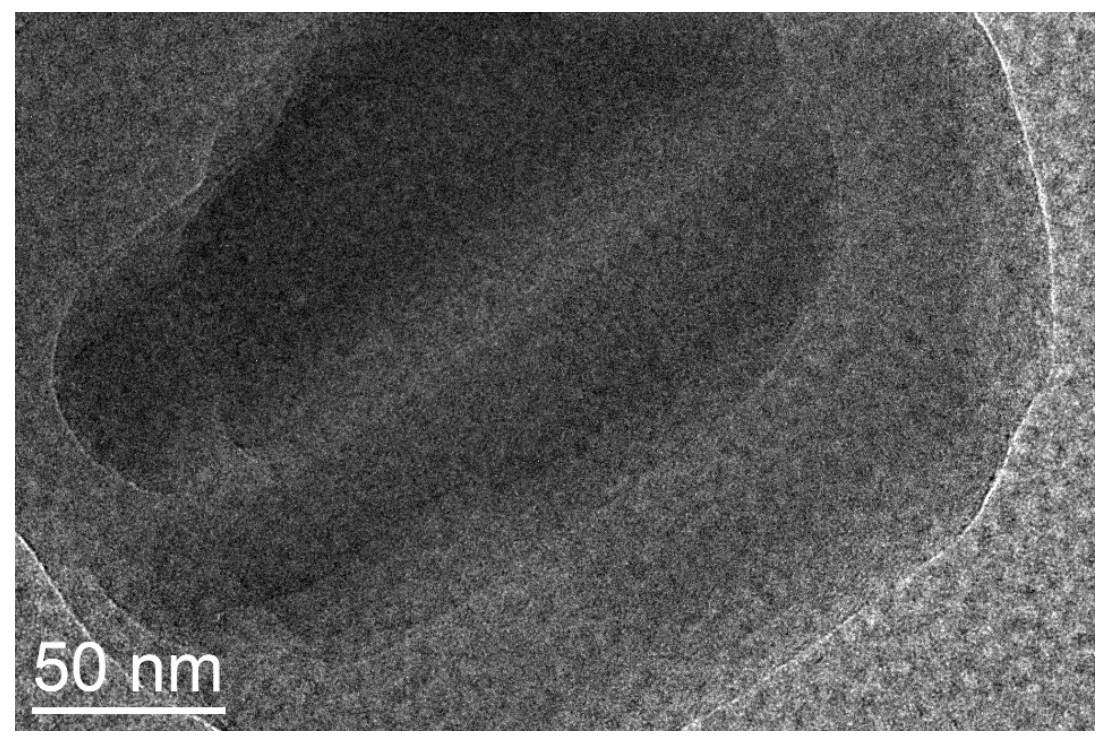

Figure S2. TEM image of $\mathrm{Rh}_{2} \mathrm{O}_{3} @ \mathrm{~S}-1$ sample. 


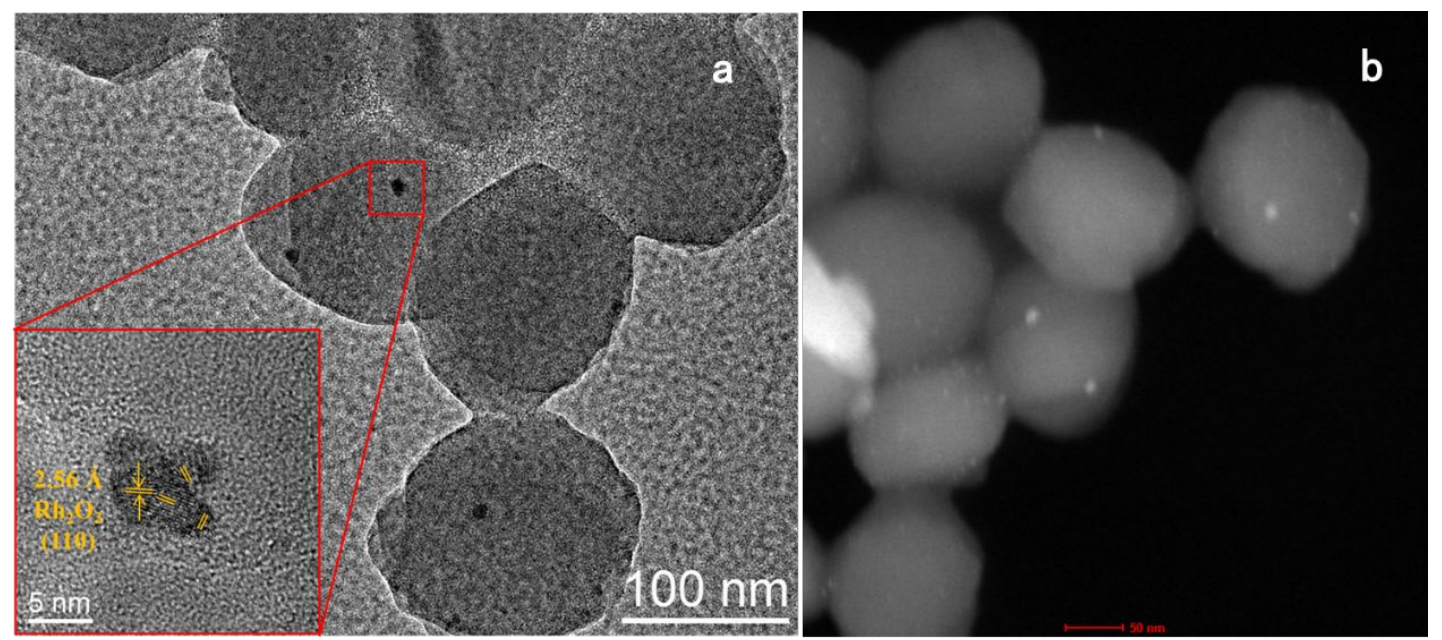

Figure S3. (a) TEM and (b) HAADF-STEM images of $\mathrm{Rh}_{2} \mathrm{O}_{3} / \mathrm{S}-1$

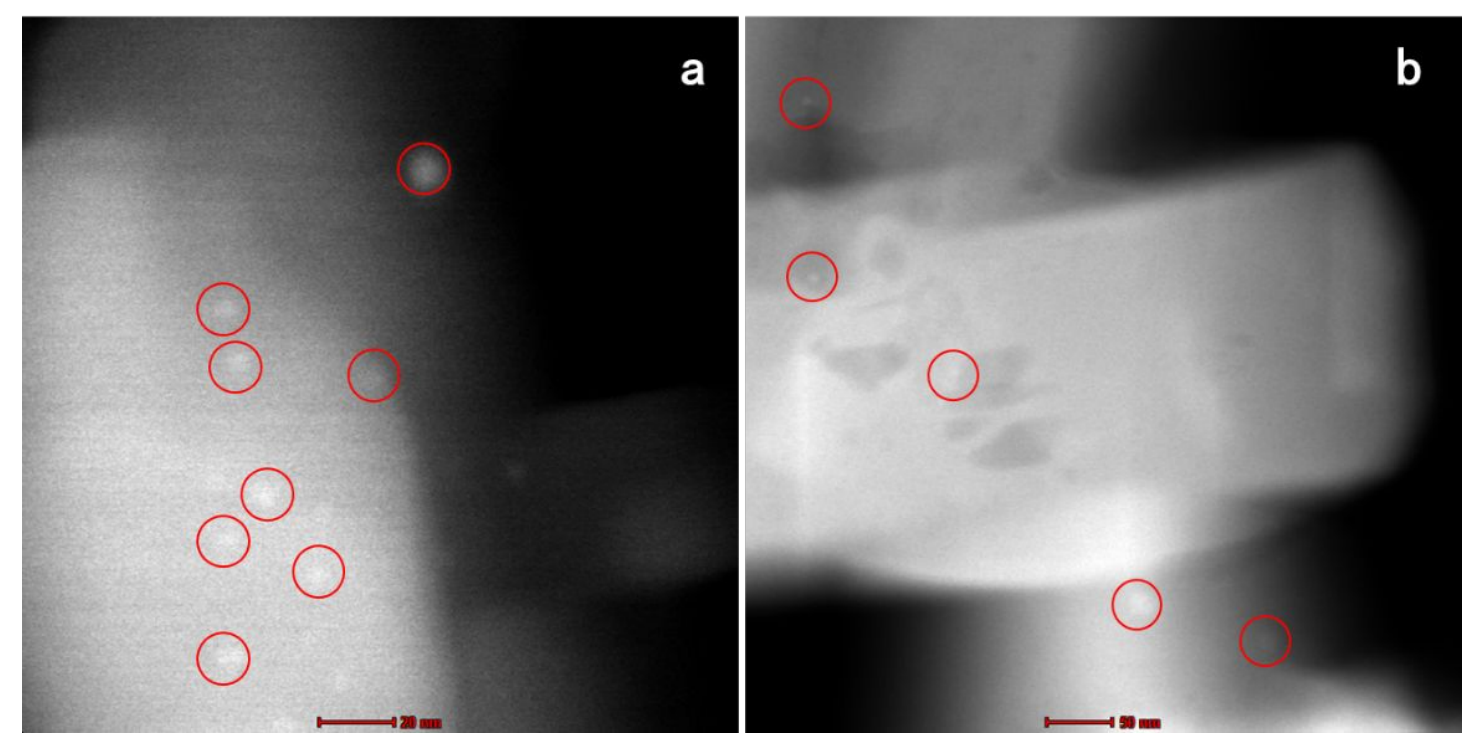

Figure S4. HAADF-STEM images of (a) $\mathrm{Rh}_{2} \mathrm{O}_{3} @ \mathrm{~S}-1$ and (b) $\mathrm{Rh}_{2} \mathrm{O}_{3} @ \mathrm{~S}-1-\mathrm{I}$ samples. The rhodium oxide nanoparticles were marked by red circles in both samples. 

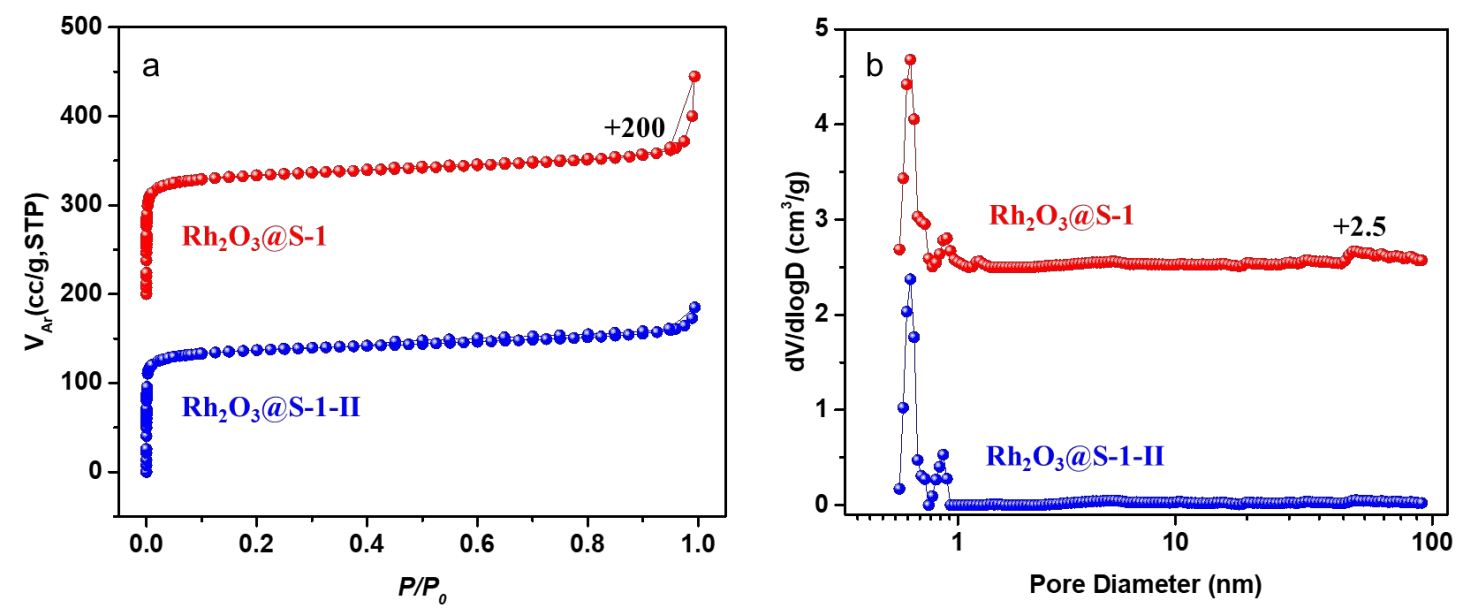

Figure S5. (a) Ar sorption isotherms at $87 \mathrm{~K}$ and (b) pore size distributions of $\mathrm{Rh}_{2} \mathrm{O}_{3} @ \mathrm{~S}-1$ and $\mathrm{Rh}_{2} \mathrm{O}_{3} @ \mathrm{~S}-1$ - II samples which were determined by non-local density functional theory (NLDFT).
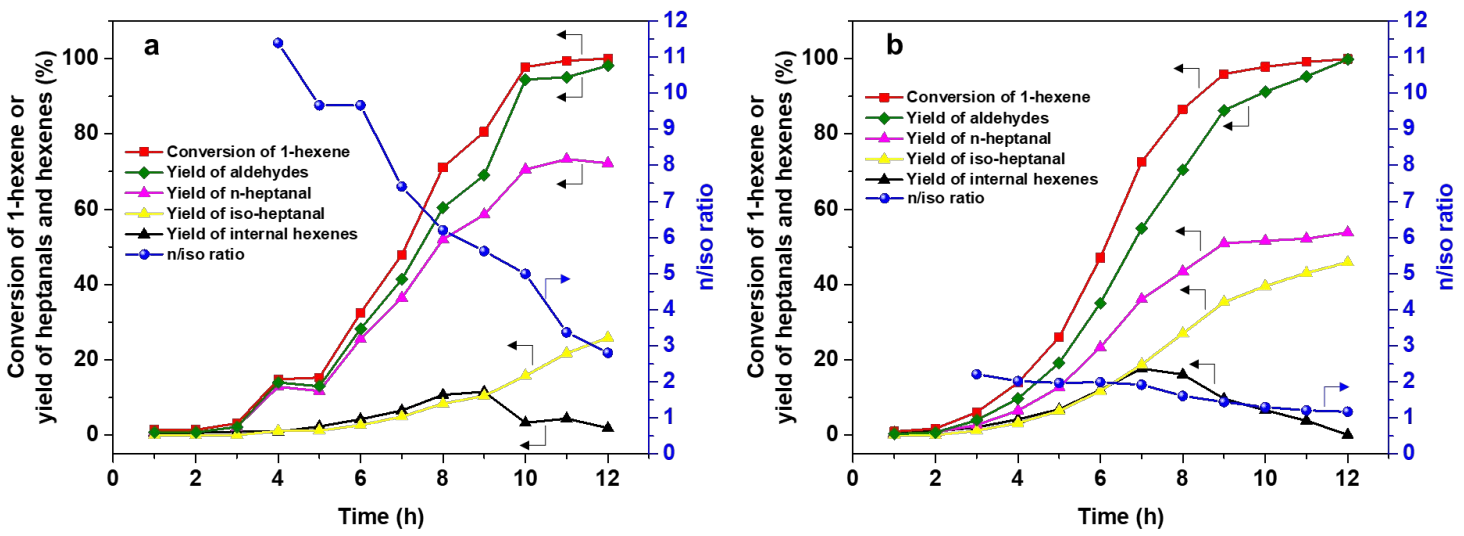

Figure S6. Dependence of the catalytic conversions, yields and regioselectivities on the reaction time over (a) $\mathrm{Rh}_{2} \mathrm{O}_{3} @ \mathrm{~S}-1-\mathrm{II}$ and (b) $\mathrm{Rh}_{2} \mathrm{O}_{3} / \mathrm{S}-1$ (reaction conditions: molar ratio of olefin to rhodium is $6400, \mathrm{CO} / \mathrm{H}_{2}=1: 1,5 \mathrm{MPa}, 10 \mathrm{~mL}$ toluene as solvent, $\left.60^{\circ} \mathrm{C}\right)$. 

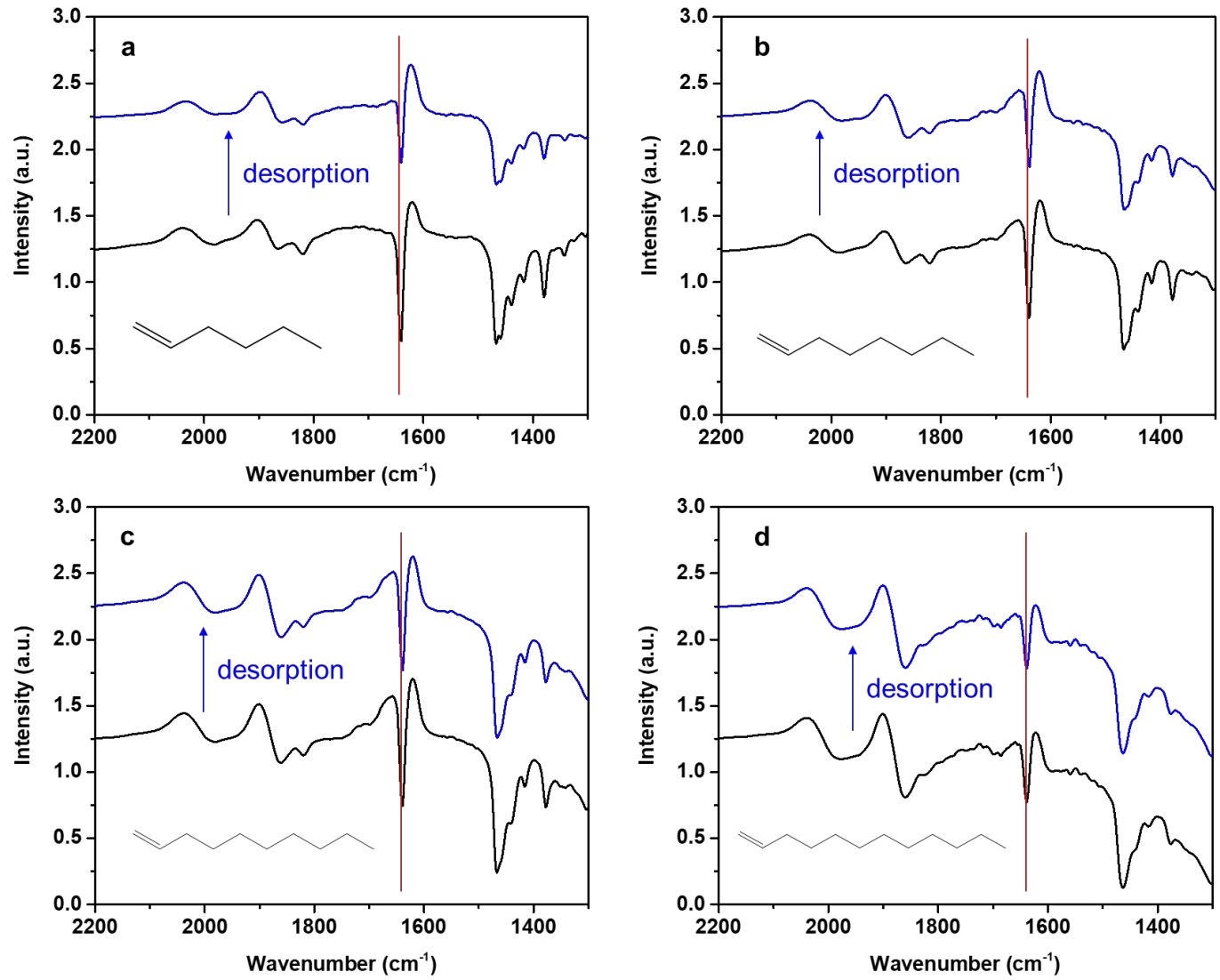

Figure S7. FT-IR spectra of (a) 1-hexene, (b) 1-octene, (c) 1-decene and (d) 1-dodecene before and after desorption on the $\mathrm{Rh}_{2} \mathrm{O}_{3} @ \mathrm{~S}-1-\mathrm{II}$.

Table S1. Rh contents in $\mathrm{Rh}_{2} \mathrm{O}_{3} / \mathrm{S}-1, \mathrm{Rh}_{2} \mathrm{O}_{3} @ \mathrm{~S}-1$ and $\mathrm{Rh}_{2} \mathrm{O}_{3} @ \mathrm{~S}-1$ - II before and after 7 recycling runs based on ICP-AES.

\begin{tabular}{ccccc}
\hline $\begin{array}{c}\text { Catalysts before } \\
\text { using }\end{array}$ & $\begin{array}{c}\text { Rh content } \\
(\mathbf{\%})-\mathbf{w}_{\mathbf{1}}\end{array}$ & $\begin{array}{c}\text { Catalysts after 7 } \\
\text { runs }\end{array}$ & $\begin{array}{c}\text { Rh content } \\
\mathbf{( \% )}-\mathbf{w}_{\mathbf{2}}\end{array}$ & $\begin{array}{c}\text { Leaching ratio } \\
(\mathbf{\%})^{\boldsymbol{a}}\end{array}$ \\
\hline $\mathrm{Rh}_{2} \mathrm{O}_{3} / \mathrm{S}-1$ & 0.15 & $\mathrm{Rh}_{2} \mathrm{O}_{3} / \mathrm{S}-1$ & 0.03 & 80 \\
$\mathrm{Rh}_{2} \mathrm{O}_{3} @ \mathrm{~S}-1$ & 0.06 & $\mathrm{Rh}_{2} \mathrm{O}_{3} @ \mathrm{~S}-1$ & 0.01 & 83 \\
$\mathrm{Rh}_{2} \mathrm{O}_{3} @ \mathrm{~S}-1-\mathrm{II}$ & 0.02 & $\mathrm{Rh}_{2} \mathrm{O}_{3} @ \mathrm{~S}-1-\mathrm{II}$ & 0.01 & 50 \\
& & & \\
${ }^{a}$ Leaching ratio: calculated by $\left(\mathrm{w}_{1}-\mathrm{w}_{2}\right) / \mathrm{w}_{1} \times 100 \%$ & \\
\hline
\end{tabular}

